\title{
Testosterone, Coronary Artery Disease, and the Syntax score in males: A case control study
}

\author{
Sezavar $\mathrm{SH}^{1}$, Hassanzadeh $\mathrm{M}^{3 *}$, Samadanifard $\mathrm{SH}^{2}$ and Zandi Karimi $\mathrm{F}^{3}$ \\ ${ }^{1}$ Department of Cardiology, Rasoul-e-Akram Hospital, Iran University of Medical Sciences, Tehran, Iran \\ ${ }^{2}$ Department of Endocrinology, Rasoul-e-Akram Hospital, Iran University of Medical Sciences, Tehran, Iran \\ ${ }^{3}$ Department of Internal Medicine, Rasoul-e-Akram Hospital, Iran University of Medical Sciences, Tehran, Iran
}

\begin{abstract}
Objectives: To determine the possible association between serum testosterone levels and the presence or severity of coronary artery disease (CAD) among male patients.

Methods: During a 6-month course, all consecutive male individuals younger than 55 years referred for angiography to the Rasoul-e-Akram General Hospital and the Day Clinic, Tehran, Iran, were recruited to the study. Excluded were those with recent history of acute coronary syndrome, history for hypogonadism, or any other medical backgrounds that interfere with results of serum testosterone level. After sending plasma samples, all individuals underwent angiography according which they were categorized as the CAD group ( $>50 \%$ occlusion) and the non-CAD group ( $<50 \%$ occlusion). Furthermore, the Syntax score was calculated for patients of the CAD group.

Results: A total of 43 patients were recruited to the study among whom 21 were categorized as the CAD group and 22 as the non-CAD one. Serum level of free testosterone was lower $(\mathrm{P}=0.010)$ and that of albumin was higher $(\mathrm{P}=0.031)$ in the $\mathrm{CAD}$ group when compared to the non-CAD one. However, the total testosterone and the bioavailable testosterone levels were not different between the two groups. No statistically significant correlation was found between the free or total testosterone levels and the Syntax score in CAD patients.
\end{abstract}

Conclusion: Male patients with CAD had lower free testosterone levels when compared to the non-CAD ones. However, the severity of CAD, determined by the Syntax score, did not correlate with the serum testosterone level. Additional studies are needed to clarify the latter issue.

Abbreviations and Symbols: CAD: Coronary Artery Disease; DM: Diabetes Mellitus; HTN: Hypertension; HLP: Hyperlipidemia; PCI: Percutaneous Coronary Intervention; FSH: Follicle-Stimulating Hormone; LH: Luteinizing Hormone; SHBG: Sex-Hormone Binding Globulin; IRMA: Immunoradiometric Assay; SD: Standard Deviation; IQR: Interquartile Range

\section{Introduction}

Coronary artery disease (CAD) remains the leading cause of death worldwide and is responsible for nearly $40 \%$ of all deaths, either directly or indirectly [1,2]. In addition, about 90 million working days each year are lost because of CAD which inflicts heavy economic costs and makes CAD one of the main causes of morbidity in all countries $[3,4]$. That is why CAD has been the subject of several preventive efforts as well as many investigations around the world.

After the well-known Framingham study [5], the CAD risk factors have been targeted in extensive medical research for many years. During the time, the role of many factors such as diabetes mellitus (DM), hypertension (HTN), hyperlipidemia (HLP), and cigarette smoking have been established as predisposing factors for CAD [6]. At the same time, however, there are some other factors that have never been established either as a risk factor or as a protective one for CAD; among which the relationship between serum levels of androgens and CAD has been a controversial debate among researchers [7-10]. Whereas some investigations reported no relationship between severity of CAD and serum androgen levels [11], some others found a positive association between either the CAD risk factors (such as obesity) [12] or coronary artery blood flow [13] with serum androgen levels.

The gold standard method for diagnosing CAD is coronary angiography that also could have a therapeutic role through percutaneous coronary intervention (PCI). The Syntax (Synergy between PCI with Taxus and cardiac surgery) system is an angiographic tool to grade the complexity of CAD and to obtain evidence-based guidelines for selecting the optimal technique of revascularization. In addition, it will allow comparison of CAD complexity in individual patients [14]. Utilizing the Syntax score as an accurate scale for CAD can be beneficial to quantitatively evaluate the impact of specific risk factors on the severity of CAD.

To more investigate the role of serum testosterone level on the development and severity of CAD, we designed this study with a novel use of the Syntax score as a quantitative measure.

Correspondence to: Morteza Hassanzadeh, MD, Internist, Department of Internal Medicine, Rasoul-e-Akram Hospital, Iran University of Medical Sciences, Tehran, Iran; Email: hassanzadeh.m@iums.ac.ir

Key words: coronary artery disease, testosterone, Syntax score

Received: March 01, 2018; Accepted: March 14, 2018; Published: March 18 2018 


\section{Materials and methods}

\section{Study participants}

Study subjects were selected from male patients referred for angiography to the Rasoul-e-Akram General Hospital and the Day Clinic, Tehran, Iran. All male individuals younger than 55 years were recruited to the study. Our exclusion criteria were:

1. History of acute coronary syndrome within the preceding 3 months

2. Any past history for hypogonadism, hormone replacement therapy, or illicit use of androgenic-anabolic steroids

3. Presence of active neoplasm, or any history of chemotherapy for malignancy

4. Known cases of hepatic cirrhosis, chronic kidney disease, or endstage renal disease

5. Any known medical illness (or taking any known medication) that could affect sex hormone levels; such as sickle cell anemia, celiac disease, or taking spironolactone, ketokonazole, etc.

\section{Study protocol}

During the 6-month course of the study, blood samples from all consecutive subjects were taken before the angiography. The samples were measured for the serum levels of total testosterone, free testosterone, follicle-stimulating hormone (FSH), luteinizing hormone (LH), sex-hormone binding globulin (SHBG), and albumin. The Elecsys reagent kits (Roche, USA) were used for the measurement of total testosterone, free testosterone, FSH and $\mathrm{LH}$; and the immunoradiometric assay (IRMA) kit (Gentaur, USA) was used for the measurement of SHBG. All assays were done in the Rasoul-e-Akram central laboratory. Other information such as age, family history for premature ischemic heart disease $(<55$ and $<65$ years in first-degree male and female relatives, respectively), history of DM, HTN, HLP, and cigarette smoking were collected into a checklist.

After sending the samples and before the results are available, a single interventional cardiologist (SHS) performed coronary angiographiy for all study patients. According to the results of angiography, participants were divided into two groups:

1. The CAD group that were defined as those with at least $>50 \%$ occlusion in one of the 3 principal coronary arteries or the left main coronary artery.

2. The non-CAD group with lesions making less than $50 \%$ occlusion.

Then the Syntax score was calculated for patients in the CAD group. The scoring system consists of the dominance of right or left coronary arteries, the total number of lesions, the vessel segments involved per lesion, the amount of the diameter reduction by the specific lesion, the presence or absence of total occlusion, trifurcation, bifurcations, aortoosteal stenosis, severe tortousity, thrombosis, heavy calcification, or diffuse disease in small vessels, and the occlusion length [15]. The final score is represented as a number, which higher values indicate more severe CAD.

\section{Ethical issues}

The ethical committee of Tehran University of Medical Sciences (TUMS) approved the study protocol. All patients were informed about the presence of the study; no additional fees were surcharged and signed consents were taken. Furthermore, all information was kept confidential and none of the information was released individually.

\section{Statistical analysis}

Data were analyzed by the software SPSS version 20.0. All quantitative values were tested for normality by the Shapiro-Wilk test [16]. The mean \pm standard deviation (SD) and the median with interquartile range (IQR) were used to express data with and without normal distribution, respectively. Qualitative data were expressed as percentages. For further analysis, independent sample t-test (for comparing normally distributed means), chi2 and Fisher-exact tests (for comparing percentages), Mann-Whitney U-test (for comparing non-normally distributed means), and Pearson correlation coefficient (to find any correlation between quantitative variables) were executed. A P-value less than 0.05 was considered as statistically significant.

\section{Results}

At the end of the study a total of 43 patients were recruited among whom 21 were categorized as the CAD group and 22 as the non-CAD group. Median (IQR) for age of the study subjects was 46 (40-52) years, and $100 \%$ of them were males as planned.

\section{Between-group comparisons}

Table 1 summarizes baseline demographic data compared between the two study groups. As seen, $17(80.9 \%)$ and $3(13.6 \%)$ patients in the CAD and the non-CAD groups had hyperlipidemia, respectively $(\mathrm{P}<0.001)$. No other statistically significant difference was found in the demographics.

After laboratory evaluation, values of serum albumin, FSH, and LH were higher in the $C A D$ group with statistical significance $(\mathrm{P}=0.031$, $\mathrm{P}=0.001$, and $\mathrm{P}=0.003$, respectively). In contrast, serum levels of free testosterone were significantly lower in the CAD group, when compared to the non-CAD one $(\mathrm{P}=0.010)$. However, no statistically significant difference was detected for levels of SHBG as well as the sum of free testosterone and the albumin-bound testosterone, namely "bioavailable testosterone", between the two groups. Table 2 represents hormone profile and other laboratory tests in the two study groups.

\section{Intra-group analyses of the $\mathrm{CAD}$ patients}

In the CAD group, the mean (+ SD) of the Syntax score was 16.33 \pm 9.63 . For further analysis, we divided the patients of the CAD group into the two age subgroups with a 50-year-old cut-point. No statistically significant difference was found in all measured factors (table 3). For example, the mean Syntax score was $16.71 \pm 9.58$ and $15.44 \pm 10.29$ for patients younger and older than 50 years, respectively $(\mathrm{P}=0.747)$. Other results are shown in the Table 3.

There was no correlation between the serum levels of total testosterone $(\mathrm{R}=0.035, \mathrm{P}=0.096)$ and free testosterone $(\mathrm{R}=0.187$, $\mathrm{P}=0.114$ ) with the Syntax score among the whole CAD patients. Similar results were detected when we looked for such a correlation in either the subgroups:

Table 1. Baseline characteristics of the study patients by their groups

\begin{tabular}{|c|c|c|c|}
\hline & CAD group (N=21) & $\begin{array}{c}\text { Non-CAD group } \\
(\mathrm{N}=22)\end{array}$ & $\boldsymbol{P}$ \\
\hline Age(years) & $47(42-52)$ & $45(38-52)$ & 0.843 \\
\hline Diabetes mellitus $^{* *}$ & $18(85.7)$ & $20(91)$ & 0.898 \\
\hline Hypertension $^{* *}$ & $17(81)$ & $18(81.8)$ & 0.990 \\
\hline CAD family history $^{* *}$ & $16(76)$ & $12(54.5)$ & 0.176 \\
\hline Smoking $^{* *}$ & $6(28.5)$ & $4(18.1)$ & 0.549 \\
\hline Hyperlipidemia $^{* *}$ & $17(80.9)$ & $3(13.6)$ & $<0.001$ \\
\hline
\end{tabular}

$*$ Value is represented as median (interquartile range)

$* *$ Values are the number $(\%)$

CAD: Coronary Artery Disease 
Table 2. Results of laboratory measurements by the study groups

\begin{tabular}{|c|c|c|c|}
\hline & CAD group & Non-CAD group \\
\hline Total Testosterone $(\mathrm{ng} / \mathrm{dl})^{*}$ & $3.22(1.81-4.63)$ & $4.33(1.45-7.21)$ \\
\hline Free testosterone(ng/dl) & $0.08(0.02-0.14)$ & $0.15(0.05-0.25)$ \\
\hline Bioavailable Testosterone(ng/dl) & $41.10(33.83-48.37)$ & $43.20(28.90-57.50)$ \\
\hline Albumin(mg/dl) & $4.59 \pm 0.33$ & $4.36 \pm 0.45$ \\
\hline Sex-Hormone Binding Globulin $(\mathrm{nmol} / \mathrm{L})^{* * *}$ & $43.73 \pm 13.90$ & $45.66 \pm 23.83$ \\
\hline Follicle-Stimulating Hormone $(\mathrm{ng} / \mathrm{dl})^{* * *}$ & $7.16 \pm 3.76$ & $4.53 \pm 1.79$ \\
\hline Luteinizing Hormone $(\mathrm{ng} / \mathrm{dl})^{*}$ & $6.26(3.28-9.24)$ & 0.477 \\
\hline
\end{tabular}

*Values are the median (interquartile range)

**Values are the mean (standard deviation)

CAD: Coronary Artey Diseas

Table 3. Results of laboratory measurements comparing two age sub-groups within the group of patients with coronary artery disease *

\begin{tabular}{|c|c|c|c|}
\hline & $\begin{array}{c}\text { Younger than 50 years } \\
(\mathbf{N = 9 )}\end{array}$ & $\begin{array}{c}\text { Older than 50 years } \\
\text { (N=12) }\end{array}$ \\
\hline Total Testosterone (ng/dl) & $1.85(1.45-2.25)$ & $4.36(2.91-5.81)$ \\
\hline Free testosterone (ng/dl) & $0.19(0.15-0.23)$ & $0.06(0.040 .08)$ \\
\hline Bioavailable Testosterone (ng/dl) & $14.48(11.32-17.64)$ & $14.17(9.45-18.89)$ \\
\hline Albumin (mg/dl) & $0.46(0.36-0.56)$ & $0.48(0.32-0.64)$ \\
\hline Sex-Hormone Binding Globulin (nmol/L) & $24.55(19.20-29.90)$ & 0.072 \\
\hline Follicle-Stimulating Hormone (ng/dl) & $3.88(3.04-4.72)$ & 0.824 \\
\hline Luteinizing Hormone (ng/dl) & $3.82(2.99-4.65)$ & 0.536 \\
\hline
\end{tabular}

*Values are represented as the median (interquartile range)

1. For CAD patients younger than 50 years, correlation coefficients (R) of the serum levels of total testosterone and free testosterone with the Syntax score were $0.013(\mathrm{P}=0.276)$ and $0.123(\mathrm{P}=0.251)$, respectively.

2. For CAD patients older than 50 years, correlation coefficients of the serum levels of total testosterone and free testosterone with the Syntax score were $0.111(\mathrm{P}=0.231)$ and $0.145(\mathrm{P}=0.104)$, respectively.

\section{Discussion}

Based on the results, hyperlipidemia was more frequent among the CAD patients $(80.9 \%$ vs. $13.6 \%, \mathrm{P}<0.001)$. Serum levels of the total testosterone and the bioavailable testosterone were not different between the two groups of patients, the CAD group and the non-CAD group. However, the serum levels of free testosterone and albumin were lower $(\mathrm{P}=0.010)$ and higher $(\mathrm{P}=0.031)$ in the CAD group than the nonCAD group, respectively. Finally, no statistically significant correlation was found between the levels of serum free or total testosterone and the Syntax score, both in all patients of the CAD group and each of its subgroups.

As a traditional risk factor for CAD [17,18], hyperlipidemia is predictable to be more frequent in the CAD group. Fortunately, it has nothing to do with the serum testosterone level and here is not considered as a possible confounding factor.

According to the literature, $\mathrm{CAD}$ is approximately twice more frequent among males than females [19-21]. That observation could lead to the assumption that serum testosterone level has a noxious effect on the cardiovascular system. Despite this, in our study the serum level of free testosterone was lower in the CAD group; a finding that has also been reported by many other investigators [22-25]. Moreover, some studies have demonstrated that testosterone levels are inversely related to the surrogate markers of atherosclerosis [26,27]. Therefore, our results in accordance with those studies suggest a testosterone deficient state might play a predisposing role for CAD in males.

The finding that free testosterone level differs between the two groups, but the level of total testosterone does not so, highlights a well- known endocrinology fact: the unbound form of different hormones is the main pathophysiologically active form $[28,29]$. The statistically significant higher levels of pituitary hormones, FSH and LH, among the CAD group can be similarly described. However, in contrast to the differences found for the hormones, the difference of about $0.3 \mathrm{mgr} /$ $\mathrm{dl}$ in serum albumin level between the two groups, although found statistically significant, is not considered as clinically meaningful. It becomes more trivial with considering the fact that there was no statistically significant difference between the two groups in the levels of the bioavailable testosterone (the sum of free testosterone and albumin-bound testosterone).

In an attempt to quantitatively investigate a correlation between the serum testosterone level and the extent of coronary damage in patients with CAD, we used the Syntax score for evaluation. Previously, $\mathrm{Li}$, et al. [30] had used the Gensini score for the severity of CAD and had found it well correlated with the testosterone level. Accordingly, we hypothesized that higher Syntax score might also correlate with the lower testosterone level. Although we could not find any statistically significant such a correlation, it might be for the low number of the recruited subjects. In order to eliminate the confounding effect of aging, another analysis among the two subgroups of CAD patients (younger and older than 50 years) also failed to demonstrate any such a correlation. More studies with higher sample sizes are needed to clarify that issue.

In this study inter-observer variability was omitted by having all angiographic evaluations performed by a single cardiologist. Moreover, to the knowledge of the authors, this is the first study that introduces the Syntax score for finding a more quantitative correlation between the severity of CAD and the testosterone level; a feature that makes our investigation unique. The main limitation of the present study, however, is the small sample size which underestimates the random effect; it also diminishes the sufficient statistical power required for obtaining all potential significant correlations. Additionally, not including males older than 55 years limits the external validity of our study.

In summary, we studied 43 male patients of whom 21 had CAD and 22 had not. In the CAD group, the serum free testosterone level 
was significantly lower $(\mathrm{P}=0.010)$ and the $\mathrm{FSH}$ and $\mathrm{LH}$ levels were significantly higher $(\mathrm{P}=0.001$ and $\mathrm{P}=0.003$, respectively) than the nonCAD group. No statistically significant correlation was found between the Syntax score and the free or total testosterone levels among the CAD patients.

\section{Conclusion}

In conclusion, male patients with CAD had lower free testosterone levels when compared to the non-CAD ones. However, the severity of CAD, determined by the Syntax score, did not correlate with the serum testosterone level. Additional studies are needed to clarify the latter issue.

\section{Funding Information}

All expenses of the present study were provided by a grant from Iran University of Medical Sciences.

\section{Competing Interests}

The authors declare that there is no competing interest.

\section{References}

1. Achenbach S, Kramer CM, Zoghbi WA, Dilsizian V (2010) The year in coronary artery disease. JACC Cardiovascular Imaging 3: 1065-1077. [Crossref]

2. Lawton JS (2011) Sex and gender differences in coronary artery disease. Semin Thorac Cardiovasc Surg 23: 126-130. [Crossref]

3. Leal J, Luengo-Fernandez R, Gray A, Petersen S, Rayner M (2006) Economic burden of cardiovascular diseases in the enlarged European Union. Eur Heart J 27: 1610-1619. [Crossref]

4. Mathers CD, Loncar D (2006) Projections of global mortality and burden of disease from 2002 to 2030. PLoS Med 3: e442. [Crossref]

5. Dawber TR, Meadors GF, Moore FE Jr (1951) Epidemiological approaches to heart disease: the Framingham Study. Am J Public Health Nations Health 41: 279-281. [Crossref]

6. Kannel WB, Dawber TR, Kagan A, Revotskie N, Stokes J 3rd. (1961) Factors of risk in the development of coronary heart disease-six year follow-up experience. The Framingham Study. Ann Intern Med 55: 33-50. [Crossref]

7. Ablewska U, Jankowski K, Rzewuska E, Liszewska-Pfejfer D, Hryniewiecki T (2011) A levels of endogenous gonadal hormones and their relationship with selected coronary artery disease risk factors among young women post myocardial infarction. Acta Biochim Pol 58: 385-389. [Crossref]

8. Hu X, Zhang K, Jiang H (2012) Is: testosterone or estrogen more important for male patients with coronary artery disease? Eur J Intern Med 23: e114-115. [Crossref]

9. Maas AH (2012) Coronary Artery Disease in Elderly Women: A Disturbed Relation of Estrogen with Testosterone. Cardiology 121: 247-248. [Crossref]

10. Schwarcz MD, Frishman WH (2010) Testosterone and coronary artery disease. Cardiol Rev 18: 251-257.

11. Davoodi G, Amirezadegan A, Borumand MA, Dehkori MR, Kazemisaeid A, et al. (2007) The relationship between level of androgenic hormones and coronary artery disease in men. Cardiovasc J Afr 18: 362-366. [Crossref]
12. van den Beld AW, de Jong FH, Grobbee DE, Pols HA, Lamberts SW (2000) Measures of bioavailable serum testosterone and estradiol and their relationships with muscle strength, bone density, and body composition in elderly men. J Clin Endocrinol Metab 85: 3276-3282. [Crossref]

13. English KM, Jones RD, Jones TH, Morice AH, Channer KS (2000) Aging reduces the responsiveness of coronary arteries from male Wistar rats to the vasodilatory action of testosterone. Clin Sci (Lond) 99: 77-82. [Crossref]

14. Jou YL, Lu TM, Chen YH, Sung SH, Wang KL, et al. (2012) Comparison of the predictive value of EuroSCORE, SYNTAX score, and clinical SYNTAX score for outcomes of patients undergoing percutaneous coronary intervention for unprotected left main coronary artery disease. Catheter Cardiovasc Interv 80: 222-230. [Crossref]

15. Sianos G, Morel MA, Kappetein AP, Morice MC, Colombo A, et al. (2005) The SYNTAX Score: an angiographic tool grading the complexity of coronary artery disease. EuroIntervention 1:219-227. [Crossref]

16. Royston P (1993) A pocket-calculator algorithm for the Shapiro-Francia test for nonnormality: an application to medicine. Stat Med 12: 181-184. [Crossref]

17. Expert Panel on Detection, Evaluation, and Treatment of High Blood Cholesterol in Adults (2001) Executive Summary of The Third Report of The National Cholesterol Education Program (NCEP) Expert Panel on Detection, Evaluation, And Treatment of High Blood Cholesterol In Adults (Adult Treatment Panel III). JAMA 285: 2486-2497. [Crossref]

18. Vine DL, Hastings GE (1994) Ischaemic heart disease and cholesterol. Absolute risk more informative than relative risk. BMJ 308: 1040, 1041. [Crossref]

19. The Atherosclerosis Risk in Communities (ARIC) Study: design and objectives. The ARIC investigators (1989) Am J Epidemiol 129: 687-702. [Crossref]

20. D'Agostino RB Sr, Vasan RS, Pencina MJ, Wolf PA, Cobain M, et al. (2008) General cardiovascular risk profile for use in primary care: the Framingham Heart Study. Circulation 117: 743-753. [Crossref]

21. Kannel WB, McGee D, Gordon T (1976) A general cardiovascular risk profile: the Framingham Study. Am J Cardiol 38: 46-51. [Crossref]

22. Barrett-Connor E, Khaw KT (1988) Endogenous sex hormones and cardiovascular disease in men. A prospective population-based study. Circulation 78: 539-545. [Crossref]

23. English KM, Mandour O, Steeds RP, Diver MJ, Jones TH, et al. (2000) Men with coronary artery disease have lower levels of androgens than men with normal coronary angiograms. Eur Heart J 21: 890-894. [Crossref]

24. Kabakci G, Yildirir A, Can I, Unsal I, Erbas B, et al. (1999) Relationship between endogenous sex hormone levels, lipoproteins and coronary atherosclerosis in men undergoing coronary angiography. Cardiology 92: 221-225. [Crossref]

25. Yeap BB (2010) Androgens and cardiovascular disease. Curr Opin Endocrinol Diabet Obesity 17: 269-276.

26. Phillips GB, Pinkernell BH, Jing TY (1994) The association of hypotestosteronemia with coronary artery disease in men. Arterioscler Thromb 14: 701-706. [Crossref]

27. Simon D1, Charles MA, Nahoul K, Orssaud G, Kremski J, et al. (1997) Association between plasma total testosterone and cardiovascular risk factors in healthy adult men: The Telecom Study. J Clin Endocrinol Metab 82: 682-685. [Crossref]

28. Ekins R (1990) Measurement of free hormones in blood. Endocr Rev 11: 5-46. [Crossref]

29. Faix JD (2013) Principles and pitfalls of free hormone measurements. Best Pract Res Clin Endocrinol Metab 27: 631-645. [Crossref]

30. Li L, Guo CY, Jia EZ, Zhu TB, Wang LS, et al. (2012) Testosterone is negatively associated with the severity of coronary atherosclerosis in men. Asian J Androl 14: 875-878. [Crossref]

Copyright: (C2018 Sezavar SH. This is an open-access article distributed under the terms of the Creative Commons Attribution License, which permits unrestricted use, distribution, and reproduction in any medium, provided the original author and source are credited. 